\section{Persistent blood eosinophilia and eosinophil activation marker in a severe case of esinophilic gastroenteritis associated with multiple food allergies}

DOI: $10.2478 /$ rrlm-2018-0026

\section{Dear Editor,}

We refer to an interesting paper published in your journal about eosinophil activation markers in clonal and non-clonal eosinophilia and we aim to add some correlations between clinical and laboratory parameters in a rare eosinophilic gastrointestinal disorder [1]. Blood eosinophilia is a frequent condition in medical practice, due to a broad spectrum of diseases, usually reactive to allergies, parasitic infections, malignancies or autoimmune diseases and more rarely primary or idiopathic. Despite lacking sensitivity and sensibility, blood eosinophilia can be considered an important laboratory parameter, mainly in cases with persistent high values and when increased eosinophil activation serologic biomarkers and secondary organ dysfunction can be proved. Eosinophilic disorders of the gastrointestinal tract, mainly eosinophilic gastroenteritis (EGE) are rare and heterogeneous disorders, with about 300 cases published in the literature, characterized by eosinophilic infiltration and inflammation at different levels, are frequently accompanied by blood eosinophilia [2]. Up to $70 \%$ of EGE patients have a personal history of allergic disorders such as asthma, rhinitis, food allergies and drug allergies or eczema, suggesting the role of hypersensitivity reactions in disease pathogenesis [3]. The clinical presentation of EGE may vary depending on the main segment affected and on depth and extent of bowel wall involvement. The underlining mechanism of EGE is eosinophilic infiltration and degranulation, with release of toxic mediators, such as eosinophil cationic protein (ECP), which was proposed as eosinophil activation marker and discriminatory tool for different types of eosinophilic disorders [1]. Peripheral blood eosinophilia is found in up to $80 \%$ of EGE cases and may have very high levels in serosal forms [4]. Endoscopic findings may be normal or nonspecific, including erythema, friability, erosions, ulcerations and nodularity, with the gastric antrum affected in about $70 \%$ of cases [5]. The proposed histopathologic definition of EGE is based on a density of the eosinophilic infiltration with a cut-off of $>20$ eosinophils per high power field [6].

We report a case of a 31 years old man who presented to our hospital in October 2017 for allergist evaluation of significant blood hypereosinophilia, persistent gastro-intestinal symptoms and significant weight loss, with progressive onset for almost one year. He had noticed moderate blood eosinophilia on his regular check-up two years before, but no other laboratory tests were performed, due to good health status. He had no personal history of allergies, but recently noticed symptoms aggravation after ingestion of some foods, mainly milk, eggs and wheat. Previous medical evaluation performed by the gastroenterologist in February 2017 revealed active gastritis and oesophagitis with florid eosinophilic infiltration and carpet of antral polyps. No specific treatment was given, since blood eosinophilia was considered possibly reactive to parasitic infections, but he continued to have nausea, vomiting, diarrhea, flatulence and weight loss. At hospital admission he had no relevant clinical signs, except diffuse moderate abdominal pain, with $\mathrm{BMI}=21.6 \mathrm{~kg} / \mathrm{m} 2$. Laboratory tests revealed high blood eosinophilia 5310/uL (normal range 
below 700/uL), representing $49.5 \%$, normal total white blood cell, moderate high serum total IgE, value $628 \mathrm{IU} / \mathrm{ml}$ (normal range below 100.0 $\mathrm{IU} / \mathrm{ml}$, using electrochemiluminescence method -ECLIA), decreased total serum proteins $(5.19 \mathrm{~g} /$ $\mathrm{dL}$, normal range $=6.60-8.70 \mathrm{~g} / \mathrm{dL})$, slight decrease of total serum calcium $(8.14 \mathrm{mg} / \mathrm{dL}$, normal range $=8.60-10.20 \mathrm{mg} / \mathrm{dL})$ and insufficient level of 25-hydroxycholecalciferol, value 10.96 $\mathrm{ng} / \mathrm{ml}$ (optimal range $=30-100 \mathrm{ng} / \mathrm{ml}$ ). We measured the serum level of eosinophil cationic protein (ECP), an important marker of activated eosinophils, which was very high $(>200 \mathrm{ug} / \mathrm{L}$, normal range below $13.3 \mathrm{ug} / \mathrm{L}$ ). We found significant high specific IgE against almost all essential foods using ImmunoCAP Food Allergy Assay, with cut-off below $0.35 \mathrm{kU} / \mathrm{L}$. The most relevant increased specific $\mathrm{IgE}$ values were against: cow milk (53 kU/L), wheat $(18 \mathrm{kU} / \mathrm{L})$, eggs $($ white $=13 \mathrm{kU} / \mathrm{L}$, yolk=7.5 kU/L), various meat $($ turkey $=23 \mathrm{kU} / \mathrm{L}$, chicken $=12.8 \mathrm{kU} / \mathrm{L}$, pork $=58 \mathrm{kU} / \mathrm{L}$, beef $=74 \mathrm{kU} / \mathrm{L})$, seafood $(15.4$ $\mathrm{kU} / \mathrm{L})$. Endoscopic examination of the upper gastro-intestinal tract was repeated, showing oesophageal mucosa with "crepe paper" aspect and Schatzki ring, stomach with multiple antral polyps. Biopsies showed important mucosal eosinophilic infiltration, with $>200$ eosinophils/HPF of the oesophagus, stomach and duodenum (Figure 1). Stool and blood tests for parasitic infections were negative. We considered that blood eosinophilia was reactive to eosinophilic gastroenteritis and esophagitis associated with multiple food allergies. Therapeutic approach of eosinophilic disorders of gastrointestinal tract depends on symptoms severity and are based on experts recommendations. Corticosteroids are the mainstay of therapy in patients with moderate to severe disease, due to their proved and prompt effect on eosinophilic inflammation. Budesonide is a

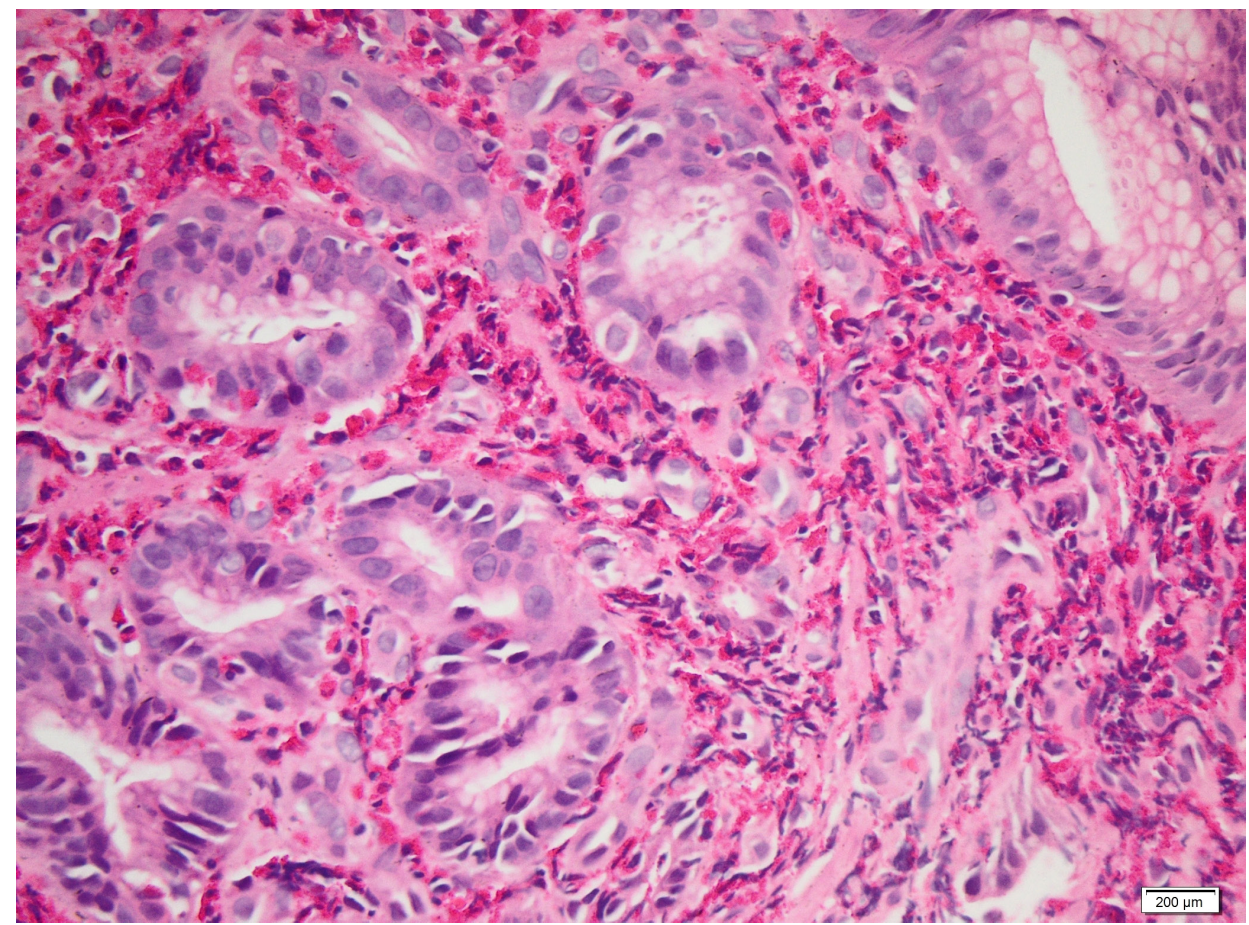

Fig. 1. Biopsy of gastric mucosa showing marked inflammation, predominantly eosinophils, >200eos/HPF, HE coloration, magnification $X 400$ 
locally acting corticosteroid with a high firstpass effect, which minimizes the systemic side effects and confers a better safety profile comparing to prednisone [7]. Duration of corticosteroid therapy is not clearly defined, depending on clinical evolution and relapses often need longterm treatment. Various steroid-sparing drugs are proposed, such as sodium cromoglycate - a stabilizer of mast cell membranes, antihistamines and montelukast - a selective leukotrienes antagonist. We first recommended elimination diet, which was difficult, due to multiple food allergies, therefore we initiated corticosteroid treatment with budesonide non-enteric-coated capsules $9 \mathrm{mg}$ daily, plus sodium cromoglycate, proton pomp inhibitors, antihistamines, calcium and vitamin D3. The clinical outcome after two months was good, with persistence of minimal gastrointestinal symptoms whereas hematologic control showed significant decrease of blood eosinophilia to $1430 / \mathrm{uL}$, increase of serum protein, calcium and $25-\mathrm{OH}$ vitamin $\mathrm{D}$ up to normal values, but ECP remained almost unchanged. We took into consideration the conclusions of the paper already mentioned [1], regarding the discriminatory role of ECP between clonal and reactive types of eosinophilia, with higher ECP serum levels in clonal eosinophilia and also the possibility of associated primary and reactive eosinophilia. We performed bone marrow examination, which showed reactive eosinophilia, with no blasts or atypic cells and we determined the genetic mutation platelet-derived growth factor receptor alpha - PDGFRA/ fibroblast growth factor receptor 1- FGFR1 (F/P) characteristic for myeloproliferative subtype of primary hypereosinophilic syndrome (HES), which was negative. The clinical evolution after one month was good, but eosinophils count came to high levels $(4140 / \mathrm{uL})$, probably due to budesonid dose reduction and incidental introduction of allergenic foods. We recommended increase of budesonide dose to $9 \mathrm{mg}$ daily, a more strict elimination diet for minimum one month and follow-up based on regular hematologic laboratory control, clinical monitoring and endoscopic evaluation after six months. Possible complications due to EGE and allergies are: malabsorbtion, severe anemia, osteoporosis, gastric or intestinal obstruction and perforation, pyloric stenosis, pancreatitis, more severe allergies, such as anaphylaxis. The natural history of EGE is not well documented and prognosis depends on risk factors, triggers, patient compliance and education. Three longterm progression patterns were described, with non-relapsing disease in the serosal type, relapsing - remitting disease in the muscular type and persistent disease predominantly observed in the mucosal type. High blood eosinophilia at diagnosis is considered an independent predictor of relapses [6].

The reported case is illustrative for difficulties in the evaluation and management of eosinophilia due to EGE and multiple food allergies in clinical practice. It also underlines the importance of correlation between clinical and laboratory parameters and the need of biomarkers for monitoring disease activity. EGE diagnosis requires a high index of suspicion, generally suggested by persistent gastrointestinal symptoms, less responsive to usual therapy and associated blood eosinophilia, after exclusion of other possible causes [8]. The main differential diagnoses of blood hypereosinophilia associated with gastrointestinal symptoms are: parasitic infections, coeliac disease, gastric cancer, lymphomas, protein-losing enteropathy, mastocytosis, vasculitis phase of Churg-Strauss syndrome and HES. This last clinical entity has no allergic pattern, is rarely accompanied by gastrointestinal involvement, but other organs, such as lungs and cardio-vascular system can be affected [9]. Food allergies represent an important risk factor and trigger for EGE, since food allergens can cross the intestinal mucosa and induce allergic inflammation, consisting in degranulation of mast cell and eo- 
sinophils. Food allergies represent an increasing pathology worldwide in both children and adults, affecting up to $10 \%$ of population and the most allergenic foods are cow's milk, hen's egg, peanuts, tree nuts and seafood [10]. Allergic diseases are usually accompanied by blood eosinophilia, usually in moderate range and this can be asymptomatic for variable periods of time. The preclinical phase of eosinophilia may suggest the period of hypersensitization to food allergenes, before clinically manifested food allergies, with severe forms in rare cases, such as EGE. In this context, blood eosinophilia may not be considered a trivial laboratory parameter and early evaluation and identification of causal disease could improve medium and long-term prognosis. Even though blood eosinophilia may not correlate with eosinophilic organic disorder, in some cases, such as the reported one, the clinical diagnosis was delayed with almost one year after the onset of progressive gastrointestinal and general symptoms accompanied by increasing eosinophilia. In this particular case, persistent blood eosinophilia could have served as an early laboratory parameter able to prompt the physician to search food allergies and to confirm EGE in an early stage. The usefulness of serum eosinophilic activation markers such as ECP in different types of eosinophilic disorders is an important topic and it needs further evaluation.

\section{Polliana Mihaela Leru ${ }^{1,2^{*}}$, Vlad Florin Anton ${ }^{1}$, Horia Zacheu ${ }^{1}$, Theodor Voiosu ${ }^{1,2}$, Dumitru Matei $^{2}$}

1. Colentina Clinical Hospital, Carol Davila University of Medicine and Pharmacy, Bucharest, Romania

2. Carol Davila University of Medicine and Pharmacy

\section{Corresponding author}

Polliana Mihaela Leru, e-mail: polianaleru@yahoo.com
Received: $3^{\text {rd }}$ April 2018

Accepted: $5^{\text {th }}$ July 2018

Published: $12^{\text {th }}$ July 2018

\section{Conflict of interest}

The authors declare that they have no conflict of interest relating to this work and manuscript.

\section{Patient consent for publication}

A copy of the patient written informed consent is available for review by the Chief Editor of this journal.

\section{References}

1. Angelescu S, Mambet C, Mut Popescu DI, Berbec N, Costache A, Isaroiu $\mathrm{M}$, et al. Eosinophil activation markers in clonal and non-clonal eosinophilia. Rev Romana Med Lab. 2013;21(3):311-20. DOI: 10.2478/ rrlm-2013-0024

2. Chen MJ, Chu CH, Lin SC, Shih SC, Wang TE. Eosinophilic gastroenteritis: Clinical experience with 15 patients. World J Gastroenterol 2003; 9(12): 2813-16. DOI: 10.3748/wjg.v9.112.2813

3. Oyaizu N, Uemura Y, Izumi H, Morii S, Nishi M, Hioki K. Eosinophilic gastroenteritis. Immunohistochemical evidence for IgE mast-cell mediated allergy. Acta Pathol Jpn 1985;35:759-766.

4. Khan S, Orenstein SR. Eosinophilic gastroenteritis. Therapy in practice. Pediatr Drugs 2002; 4(9):563-70. DOI: $10.2165 / 00128072-200204090-00002$

5. Ingle SB, Hinge CR. Eosinophilic gastroenteritis: An unusual type of gastroenteritis. World J Gastroenterol. 2013;19(31):5061-5066. DOI: 10.3748/wjg.v19. i3 1.5061

6. Abou Rached A, El Hajj. Eosinophilic gastroenteritis : Approach to diagnosis and management. World J Gastrointest Pharmacol Ther. 2016;7(4):513-23. DOI: 10.4292/wjgpt.v7.i4.513

7. Tan ACITL, Kruimel JW, Naber THJ. Eosinophilic gastroenteritis treated with nonenteric-coated budesonide tablets. Case report. Eur J Gastroenterol Hepatol. 2001;13:425-7. DOI: 10.1097/00042737- 


\section{0-00021}

8. Mori A, Enweluzo C, Grier D, Badireddy M. Eosinophilic gastroenteritis: Review of a rare and treatable disease of the gastrointestinal tract. Case Rep Gastroenterol 2013;7:293-8. DOI: 10.1159/000354147

9. Gotlib J. World Health Organization-defined eosinophilic disorders: 2014 update on diagnosis, risk stratification and management. Annual Clinical Up- date in Hematological Malignancies. Am J Hematol. 2014;89;326-37. DOI: 10.1002/ajh.23664

10. Sicherer SH, Allen K, Lack G, Taylor SL, Donovan SM, Oria M. Critical issues in Food Allergy: A National Academies Consensus Report. Pediatrics. 2017;140(2):e20170194. DOI: 10.1542/peds.20170194 